Itinéraires Itinéraires

Littérature, textes, cultures

2020-3 | 2021

Le rap, une poésie de performances

\title{
«Les anarchistes couronnés » : rap hardcore et droit de cité
}

"Crowned Anarchists": Hardcore Rap Music and its Rightful Space

\section{Elsa Vallot}

\section{Q OpenEdition}

1 Journals

Édition électronique

URL : https://journals.openedition.org/itineraires/8968

DOI : $10.4000 /$ itineraires.8968

ISSN : 2427-920X

Éditeur

Pléiade

Référence électronique

Elsa Vallot, " «Les anarchistes couronnés » : rap hardcore et droit de cité », Itinéraires [En ligne], 2020-3 | 2021, mis en ligne le 08 décembre 2021, consulté le 12 décembre 2021. URL : http:// journals.openedition.org/itineraires/8968; DOI : https://doi.org/10.4000/itineraires.8968

Ce document a été généré automatiquement le 12 décembre 2021.

\section{(c) (i) (9)}

Itinéraires est mis à disposition selon les termes de la licence Creative Commons Attribution - Pas d'Utilisation Commerciale - Pas de Modification 4.0 International. 


\title{
« Les anarchistes couronnés » : rap hardcore et droit de cité
}

"Crowned Anarchists": Hardcore Rap Music and its Rightful Space

\author{
Elsa Vallot
}

« Et l'anarchie, au point où Héliogabale la pousse, c'est de la poésie réalisée. Il y a dans toute poésie une contradiction essentielle. La poésie, c'est de la multiplicité broyée et qui rend des flammes. Et la poésie, qui ramène l'ordre, ressuscite d'abord le désordre, le désordre aux aspects enflammés ; elle fait s'entrechoquer des aspects qu'elle ramène à un point unique : feu, geste, sang, cri. » Antonin Artaud, Héliogabale ou l'anarchiste couronné, 1979.

«J'ai la grosse tête la couronne me va pas, j'la porte à l'auriculaire "

Lino, « Suicide commercial », Requiem, 2014.

Héliogabale est l'empereur poète qui utilisa la poésie pour gouverner. Il est pour Artaud la figure de "l'anarchiste couronné ", refusant les règles de la cité tout en instituant ce refus en ordre politique ([1979] 2017 : 97). Selon Artaud, l'anarchie en poésie produit un point de feu, de geste, de sang et de cris, un acte de "poésie réalisée ", où le champ de l'imaginaire et de l'esthétique rejoint la matérialité du corps. Ce point de rencontre défini par la poésie d'Héliogabale, poésie de la performance, de la transgression et de la construction d'une figure souveraine avant tout, présente un cadre sémantique, esthétique et politique utile pour comprendre les formes et la portée du rap dans la représentation, la sémantique, et l'imaginaire. En effet, une poésie réalisée sous le signe de l'anarchie pourrait bien se situer dans la performance de rap où le corps produit et représente un signifiant poétique à la fois porté sur le son et le sens, où le corps est mis au centre, en tant qu'objet, outil, matière et vecteur de pensée. Comme le suggère 
l'adjectif "hardcore" - une matière dure, compacte, brute-, l'esthétique dite "hardcore " opte pour une approche matérialiste et radicale du corps et de son organicité. Elle suggère également une radicalité assumée par les artistes s'identifiant à ce genre en général, et les rappeurs en particulier, via la production et la mise en circulation d'opinions tranchées, d'une rhétorique aiguisée, notamment en lien avec la pensée décoloniale. Dès lors, l'écoute d'un rap hardcore, bien qu'elle s'inscrive dans un schéma de consommation globale de la culture hip-hop et participe à la production d'un imaginaire interprétatif au sens d'Edward Saïd ([1978] 2003 : 173), propose une performance à la frontière entre l'imaginaire et le matériel, où la pensée et l'émotion se déplacent, s'affinent. L'écoute du rap hardcore interroge l'auditeur sur la dimension, la forme, le style de la performance, ainsi que sur ses effets dans le réel. Tricia Rose (1994) et Paul Gilroy (1993) ont montré que le rap aux États-Unis était une technologie d'exploration et de critique du signifiant noir, que la production culturelle de hip-hop aux États-Unis est liée à l'histoire et aux luttes de la communauté afro-américaine, et que cette production emprunte à et est imprégnée par la culture afro-diasporique. Oneka LaBennett (2011) et Bakari Kitwana (2005) ont discuté les manières dont cet héritage culturel « afro » ou « noir » était célébré ou contesté au sein de la communauté afro-américaine ainsi que la façon dont des individus extérieurs à cette communauté se l'étaient approprié. Puisqu'en France, le hip-hop fait l'objet d'une reprise et d'une variation francophone, ses formes esthétiques répondent à d'autres constructions et d'autres conceptions de l'identité noire et de la communauté afro en particulier, mais aussi d'une approche différente des concepts d'identité et de communauté. La culture afropéenne, par exemple, peut se lire comme une subculture utilisant le mouvement hip-hop pour se définir ${ }^{1}$. Dès les années 1990, le hip-hop en France ouvre la voie à différentes subcultures, dont le rap hardcore, et ce phénomène place la question du style (Hebdige 1979) au centre de notre compréhension. En 1991, le groupe Ministère A.M.E.R, créé à Sarcelle, et son album Pourquoi tant de haine (1992), conduisent à une polémique nationale et est censuré par Charles Pasqua, ministre de l'Intérieur. Avec des rappeurs tels que EJM ("Élément Dangereux», 1990), les groupes Timide et sans complexe ("Hardcore », 1995), Idéal J (« Hardcore », 1998), NTM, Secteur Ä, ou encore la Mafia K'1 Fry, ainsi que le rappeur La Hyène, des émissions spécialisées telle que Sang d'encre de Jean-Pierre Seck sur Génération 88.2, dont la compilation sortie en 1999 propose des clashs entre artistes, un mouvement de rap français se qualifiant comme hardcore émerge. Il se poursuit dans les années 2000 avec le groupe Tandem, le titre "Sois Hardcore» (2006) de Stomy Bugsy avec Lino, Mystik, Despo Rutti, T.Killa, Alpha 5.20 et James K Page ${ }^{2}$, ou encore la Mafia K'1 Fry. Ce style de rap est parfois associé à la catégorie de "rap conscient", dans la mesure où le caractère cru et direct de cette musique lui permet d'aborder des thèmes complexes, parfois polémiques. Il ouvre sur un imaginaire centré autour du corps (à la fois celui du rappeur et de l'auditeur), dès lors qu'il engendre la construction d'une persona hardcore, parfois signifiée par des accessoires permettant d'étendre la sémantique du rap au corps du rappeur : $t$-shirts à punchlines ${ }^{3}$, accessoires issus de l'univers du braquage, des arts martiaux, ou du cinéma gore ${ }^{4}$. Aussi, via la construction matérielle et sonore d'une performance radicalement intense, le plaisir musical prend une part égale au plaisir lié à la stimulation de l'imaginaire. Reconnaître la subtilité du rap hardcore, c'est comprendre alors comment nos perceptions sensibles et intellectuelles se redéfinissent par le biais d'une certaine poétique, et comment la musique oriente nos corps dans un espace sémantique et symbolique. En partant du principe que l'expérience de l'intime est traversée par le 
politique, et que, comme l'a montré Sara Ahmed (2007), les corps sont précédés par une histoire et un héritage social et politique, il est légitime de se demander dans quelle mesure le style, la poétique, les sonorités et la performance hardcore résonnent et font vibrer la sphère politique; tout comme il est légitime de se demander de quelles manières la musique de rap hardcore est traversée, parfois violemment, par le politique. La philosophe Suely Rolnik (2011), à propos de la formation du sujet, écrit que l'écoute attentive de nos "cartographies sentimentales", des émotions qui nous orientent dans un espace et qui se trouvent parfois organisées par cette espace, est porteuse d'une expérience politique sensible. À notre tour, nous utilisons les cultural studies, la critique littéraire, la pensée décoloniale et la philosophie queer pour penser l'esthétique du rap hardcore en tant que mouvement créé et renouvelé par des artistes majoritairement non blancs, masculins et issus des quartiers populaires, et nous émettons l'hypothèse que l'expérience musicale du rap hardcore en particulier contribue à l'émergence de revendications faisant désordre dans l'espace public français construit comme blanc, masculin, colonial, autoritaire ${ }^{5}$, et questionnant profondément la notion de droit de citéb. Ainsi, la question de l'appartenance à une démocratie, à un peuple, les questions liées à la légitimité du sujet politique et de la souveraineté de certains corps vis-à-vis d'autres corps sont subtilement abordées et remodelées par la musique. Les références aux lieux ainsi que l'emploi du vocabulaire et des symboles institutionnels sont autant de marqueurs du déplacement d'un plaisir purement esthétique vers le désir de faire entendre des voix dissonantes dans le débat national. Cette étude propose de penser la formation du sujet au travers de performances musicales articulant l'espace, la souveraineté et les affects. Nous avons fait le choix d'un corpus contemporain de rappeurs ayant créé et performé ensemble (lors de featurings), au même moment (entre 2015 et 2020), et revendiquant le même espace géopoétique (le département de la Seine-Saint-Denis, Paris et sa banlieue). Kalash Criminel, Fianso, Médine, et les rappeurs gravitant autour d'eux représentent selon nous, des artistes reprenant les codes de l'esthétique hardcore, y faisant référence dans leurs paroles et dans leurs gestes. Nous analyserons tour à tour les références au corps et les références à l'espace avant de proposer une réflexion sur le droit de cité du point de vue de rappeurs hardcore. Pour ce faire, nous proposons une définition sémantique et matérialiste de l'adjectif hardcore, qui n'a jusqu'ici jamais été clairement défini autrement que comme « la tendance dure » d'un mouvement musical. Nous étayons notre définition au gré de l'analyse de notre corpus, tout en utilisant une figure littéraire, celle de l'anarchiste couronné, afin de rappeler la dimension critique de notre démarche. L'approche esthétique est, quant à elle, fondamentale, puisqu'elle permet de dissocier l'objet culturel et le geste artistique d'un certain déterminisme social. Si le rap en France reste historiquement et majoritairement un objet, un mouvement et un art créé, porté et renouvelé par des individus issus des quartiers populaires, cette analyse cherche à démontrer la singularité de l'esthétique hardcore, ainsi que la manière dont circulent les formes de cette esthétique, afin de nuancer l'association trop rapidement établie entre un espace dominé, et un art perçu comme dur et violent.

\section{Le sang : s'emparer du corps, de la voix au vêtement}

2 En 2018, le sociologue Karim Hammou décrit l'imaginaire sémantique qui entoure le rap en ces termes : « le rap est défini à la lumière du paquet interprétatif du "problème 
des banlieues", un cadrage médiatique qui en fait une musique des Autres, dont l'altérité est indissociablement sociale et raciale ». À la suite de ce constat, Marion Dalibert (2018) a montré comment la médiatisation des rappeureuse's "dissociait symboliquement les artistes d'un "Nous" français ou occidental ", afin de valoriser le groupe majoritaire «en les donnant à voir comme plus égalitaires». Si ces travaux permettent de critiquer cet imaginaire figé, force est de constater que les rappeurs ont eux-mêmes cherché à déconstruire, voire à détruire les principes d'altérisation et d'antagonisme qui déterminent leur existence dans le champ médiatique. Dans les textes de rap français, la violence de la projection du signifiant «banlieue » fait l'objet de nombreuses variantes. Des émotions fortes et "choquantes" associées à un imaginaire populaire, non blanc et masculin sont reprises et réinvesties dans le champ musical, tandis que des figures marginales sont projetées sur des corps altérisés; le banlieusard, la racaille ou le lascar, émergent sous des traits singuliers et se renouvellent au cours de la performance. En ce sens, le travail artistique du rappeur sevranais Kalash Criminel (Amira Kiziamina) élabore et met en scène de façon minutieuse une persona renvoyant à l'imaginaire des quartiers populaires et du lieu de la cité, mais dont les ressorts esthétiques dévient vers une approche purement sensible du rap. Amateur de trap, un style de rap associé à la région imaginée du Dirty South états-unien ${ }^{7}$, Kalash Criminel se distingue visuellement par le port d'une cagoule ainsi que par des survêtements de sport, notamment ceux de la marque FootKorner, dont il est l'égérie. C'est lors de l'élaboration d'un personnage gore pour le visuel de son premier morceau « Traumatisé » (2012) qu'il décide d'adopter la cagoule en particulier :

Je voulais vraiment choquer, j'étais avec des potes à moi, j'étais déjà cagoulé, j'avais une tronçonneuse, j'étais dans un délire. Finalement on a sorti que le son. Après mon premier clip, j'ai rappé cagoulé, mais sans finalement me cacher ou quoi, car j'étais en mode cagoulé mais ça veut rien dire. En fait c'est un délire que j'ai pris depuis le début. Parce que moi je veux pas rapper pour être connu [...] moi je rappe parce que je kiffe le rap. Je voulais rapper cagoulé, et c'est venu comme ça. [...] ça n'a rien à voir avec le fait d'être albinos. [...] après dans le rap les gens sont encore plus intéressés : « ouais comment ça se fait, c'est un albinos, il rappe comme ça, il est aussi violent...8 "

3 La cagoule, accessoire de mode issu de l'univers des braquages, mais aussi du BDSM et des combats de catch, véhicule anonymat, opacité, et brouille le rapport à l'identification au corps. Selon Kalash Criminel, l'objet extirpe l'artiste d'une logique simpliste consistant à associer un genre violent à une identité transparente, donnée d'emblée, notamment par le signifiant de la couleur de peau. Pour lui, le choix de la cagoule articule les marqueurs d'une violence sémantique, tout autant qu'il soutient le désir de faire remonter à la surface du corps une expérience viscérale, de convoquer une radicalité opérante dans la performance hardcore. Cette radicalité peut alors se lire comme une fin en soi : «je rappe parce que je kiffe le rap». Elle place l'organique et le plaisir au centre de l'expérience esthétique. De fait, la persona de Kalash Criminel ouvre sur un univers sonore, visuel et matériel orienté vers le sujet, le corps, l'expérience de soi. Cette persona n'est pas le fruit d'une relation antagoniste ou d'un processus d'altérisation, mais résulte bien d'un rapport à soi et d'une estime de soi importante et valorisée.

4 En 2015, Kalash Criminel est invité par le rappeur Kaaris dans l'émission Planète Rap de la radio Skyrock. À cette occasion, le public de rap mainstream découvre la persona de rappeur cagoulé-Sevranais-d'origine-congolaise de Kalash Criminel, qui se démarque comme nouveau talent du rap français. Sur le plan sonore, Kalash Criminel construit sa 
persona avec ses gimmicks tels que "Sauvage ", "Sauva-g'rie ", "Africain sauvage ", «R.A.S». Sur le plan linguistique, le rappeur utilise des mots de lingala, et des morceaux comme «Coltan» (2018) et «Roi des Sauvages » (2017) font régulièrement référence à son pays d'origine pour dénoncer la situation néocoloniale de la République démocratique du Congo'. De plus, Kalash Criminel investit l'univers des sports de combat, en particulier le MMA, comme dans le morceau "Street Fight» (2021) de Tovaritch ou dans la vidéo de son freestyle « Double K» (2018) produit pour le média Rapélite. Ainsi, et pour reprendre Artaud, dénonciations, gimmicks sonores, gestes combattants, sont autant de caractéristiques qui « s'entrechoquent » et constituent une poétique hardcore centrée sur la persona de Kalash Criminel. Du fait des références à divers usages de la violence tels que les sports de combat, les guerres ou la police, le style hardcore est souvent perçu comme violent en tant que tel. En 2016, un journaliste du média Yard TV questionne ce choix esthétique de la violence en abordant la notion de réel :

On sent qu'il y a une violence textuelle rien que dans ton nom [...] et justement cette violence-là, par rapport au cadre où tu vis dans le quartier, est-ce qu'il y a un réel décalage ou c'est vraiment une réalité ? Il y a beaucoup de rappeurs qui fantasment la violence, est-ce que toi de ton point de vue tu mets la réalité un peu à plat dans tes textes ${ }^{10}$ ?

5 Ambiguë, la notion de réel marque la recherche d'une authenticité palpable au travers d'anecdotes liées à l'expérience du quotidien à Sevran. Or, c'est cette authenticité recherchée et supposée qui empêche précisément de comprendre le travail esthétique de Kalash Criminel. Ici, l'origine sevranaise d'Amira Kiziamina participe à une surenchère fantasmée du personnage de Kalash Criminel, surenchère que les journalistes s'obstinent à vouloir expliquer par sa trajectoire sociale. La même année et dans un autre média, Kalash Criminel s'exprime pour la première fois à propos des fantasmes de violence projetés sur son environnement :

On peut dire que je renforce les clichés mais après c'est pas faux... Après c'est pas qu'à Sevran, c'est-à-dire que dans tous les quartiers, tu peux pas venir comme ça, si tu connais personne, tu viens... c'est pas un musée, tu vas pas venir visiter, je te mens pas. Dans tous les quartiers c'est comme ça frère, si t'es pas invité tu viens comme ça, on va te demander t'es un mec d'où ? Tu fais quoi ici' ${ }^{11}$ ?

Dans sa réponse, Kalash Criminel propose une rhétorique construite autour de la notion d'espace, et développe une approche singulière. L'opposition entre l'espace institutionnel du musée - espace du voyeurisme où des objets sont exhibés sous vide -, et le quartier - espace de l'affect et du relationnel, voire, privé, auquel on accède sur invitation -, est en ce sens parlante. Elle suggère également que le quartier s'érige en contre-institution, à la manière d'un négatif affecté du musée. Dès lors, le quartier devient cet autre espace habité par des œuvres d'art et de culture, en l'occurrence, par les rappeurs auxquels sont liées des activités artistiques telles que la musique, le texte, la vidéo, la danse et la mode. S'imposant à nos imaginaires de façon critique, l'opposition entre le musée et le quartier telle que décrite par Kalash Criminel désigne la distance et le décalage entre les différentes représentations des lieux. Aux musées, l'art, la culture et l'histoire, aux quartiers, la violence.

Or, les espaces et les références institutionnelles foisonnent dans l'œuvre de Kalash Criminel. Elles permettent d'inverser, de déplacer, voire de mêler et de brouiller les fonctions généralement associées à certains espaces, comme dans le titre "Cougar Gang $^{12}$ ». Seul protagoniste du clip, le rappeur y joue le rôle d'un orateur situé au beau milieu d'un Palais de l'Élysée crépusculaire. Le couplet se déploie autour de la punchline 
provocatrice - «J'baise que des mères comme Macron » - qui choque au point d'être interdit de parution sur album ${ }^{13}$. Moins que l'image pornographique et incestueuse, c'est bien l'association entre le nom propre du président et un acte sexuel qui pose ici un problème moral. Dans le morceau, l'espace symbolique du pouvoir exécutif est investi par des affects intenses liés à la référence au sexe et à la bagarre, un beat de trap évoquant un héritage culturel, historique et musical afro-américain, non français, autant d'éléments qui, assemblés et mis en musique, constituent une provocation, un détournement, ainsi qu'un affront à l'image et au prestige de ce pouvoir. Aussi, on pourrait dire avec Jacques Derrida ([1968] 1972) que le morceau "Cougar Gang" engendre une "différance »; une altération de la division supposée entre la sphère du pouvoir et la sphère de la sexualité. Là où le pouvoir aurait tendance à contrôler cette sexualité par le mariage, autorisé à certain'e·s, et les restrictions de l'accès à la santé féminine pour les personnes sans-papiers, précaires, et dominées, Kalash Criminel prend place et absorbe l'aura du président en pénétrant dans l'intimité de son corps et de sa chair.

7 Ayant pour sujet le couple présidentiel, la portée critique ainsi que l'aspect hardcore du morceau créent un désordre poétique, au sens d'Artaud. Ce désordre trouve une certaine efficacité puisque le symbole de l'exécutif est dénigré dans sa dimension autoritaire, patriarcale et symbolique, tout en étant réincarné, ramené au corporel et au charnel. Aussi, l'importance accordée à la Première dame de France dans le système républicain est déconstruite. En s'invitant lui-même dans le "gang" des hommes attirés par des femmes plus âgées, Kalash Criminel rejette son état de sujétion vis-à-vis du président Macron et se place, par l'orientation de son désir, sur une ligne de ressemblance, d'équivalence, de comparaison avec lui. De fait, ce n'est plus un homme mais un gang, un collectif sexué plein d'une volonté de pouvoir anarchique qui investit l'Élysée : «ici y'a pas de boss, tout le monde porte ses ye-cou ». Cette figure d'un gang d'affranchis interroge et confronte ${ }^{14}$ l'état de la structure hiérarchique du pouvoir exécutif ainsi que les émotions gouvernant le sujet et le politique. Le désordre poétique et émotionnel créé par le morceau et la vidéo se constitue en un tout anarchique visant à la déconstruction du pouvoir. L'africanité articulée sur un mode péjoratif (" africain sauvage »), la déviance sexuelle ( $\mathrm{j}$ 'baise des mères »), ainsi que la violence symbolique de la persona de Kalash Criminel sont autant d'éléments performatifs mis au service de la célébration d'identités construites en marges: «Laissez-nous profiter, on a trop souffert / J'pense au Congo, motema pasi ».

8 Cette marginalité subversive souvent représentée par les rappeurs touche aux catégories de la race, du genre et de la classe. Elle se pose comme un système référentiel de la performance dès lors que l'identité culturelle (Hall 1989) devient un topos, sans se figer pour autant. Sur le morceau «Street Fight » (2021) en featuring avec Tovaritch, Kalash Criminel commence son rap par une punchline faisant un autre affront à la figure présidentielle. Il mobilise une figure blanche, musulmane, issue de l'univers des arts martiaux : « eh, j'te menace comme Khabib [Nurmagomedov] menace Macron ». Par cette comparaison, le rappeur se place en solidarité avec la communauté musulmane de France et d'Occident, et opère un brouillage des catégories de son identité propre dans le sens où Kalash Criminel s'inscrit aussi, et peut-être avant tout, en tant qu'artiste et penseur critique évoluant dans le paysage politique occidental.

Par ses références culturelles, ses accessoires, ses gimmicks et ses choix textuels, Kalash Criminel construit une persona hardcore et poétique. Cette persona se lit comme une 
« différance » au sens où elle opère un glissement des signifiants de l'identité et qu'elle est elle-même le résultat d'une écriture utilisant les mots mais aussi le corps pour se déployer. Comme le suggère la figure du poète-empereur, la création d'une persona esthétique forte et liée à l'identité du rappeur met en lumière la dualité de la notion de souveraineté : sont souverains celles et ceux qui se perçoivent en tant que sujets à part entière, et celles et ceux capables de construire un discours à propos et à partir de leur position.

\section{Le cri : réclamer l'espace du 93}

Le thème de la spatialité est central dans les performances de rap en général qui, comme le rappelle Séverin Guillard (2017), se construit dans un contexte local ou national au sein duquel le rappeur prend position. Edward Saïd ([1978] 2005 : 111) a également mis en lumière la dimension sensible des références littéraires de la spatialité : « la géographie et l'histoire imaginaire aident l'esprit à rendre plus intense son sentiment intime de lui-même en dramatisant la distance et la différence entre ce qui est proche et ce qui est très éloigné ». De fait, la représentation et la relation à l'espace engagent les individus dans leur intimité, et ce dans la mesure où ces représentations les poussent à se positionner, soit en tant que spectateur/auditeur/ lecteur, soit en tant qu'artiste/penseur. Aussi, lorsque le rappeur s'empare du thème de la spatialité, il enrichit l'esthétique géo-musicale du lieu (Grassy 2010) par des images sonores et affectées. Le cas du rap hardcore faisant référence à la Seine-Saint-Denis ou au « 93 » nous montre que la simple évocation du lieu se charge d'affects liés à la représentation médiatique du département, et interroge la façon spécifique de revendiquer l'espace au travers d'une esthétique hardcore. Façonnée par l'histoire des Banlieues Rouges et du mouvement migratoire des années 1970 en France, la représentation du «quatre-vingt-zetrei » dans les médias se construit comme un espace dystopique porteur de peurs sociales ${ }^{15}$, responsable des problèmes sociétaux mais aussi comme un espace exotisé, dont les enjeux politiques sont mis à distance par les médias (Bourdieu 1996, Dalibert 2020). Nous allons voir comment, selon le mouvement anarchique de la poétique d'Héliogabale, une certaine poétique du 93 fait désordre en s'insérant dans l'hic et nunc de l'enregistrement sonore, et comment cette poétique répond à un discours sur-situé, chargé de stigmates.

11 Tout d'abord, on remarque que les rappeurs qui revendiquent une appartenance au 93 se réfèrent régulièrement au gangsta rap américain afin d'imaginer le ghettocentric turn ou la gangstaisation de l'espace. Selon Baldwin (1999), dès les années 1990, les processus de marchandisation de la culture hip-hop centrée autour d'un discours sur la violence affectent la perception du rap, passant d'un art perçu comme authentiquement noir à un art perçu comme centré sur l'expérience authentique et violente d'une vie dans le "ghetto ", et où le signifiant noir n'est plus au centre du débat, bien qu'il soit alors associé à l'expérience "gangsta ». Dès lors, les critères d'authenticité du rap américain évoluent avec le gangsta rap autour d'autres identités, telles que l'identité latinx, mais aussi autour de figures de la rue, du crime, de la violence, comme le parrain de la mafia italienne blanche ou le proxénète. Paradoxalement, cet important changement permet à des artistes extérieurs à la communauté afro-américaine de participer à une scène de rap nationale et globale, mise en relation avec l'héritage historique et politique de cette communauté. En 1995, le groupe Suprême NTM collabore avec les rappeurs et 
rappeuses NAS, AZ et Foxy Brown sur un remix franco-américain du titre « Affirmative Action $^{16} »$ (1996). Le morceau apparaît en bonus sur l'album Paris sous les bombes ${ }^{17}$ (Epic Records), et est renommé "Affirmative Action (Saint-Denis Style Remix)» en 1995. Dans son ensemble, le texte sous-entend un vécu parallèle et proche entre le centre urbain de la ville de New York et la ville de Saint-Denis. Il fait émerger une narration du quotidien centrée autour des problèmes liés au trafic de drogue, présente les rappeurs du groupe NTM (Joey Starr et Kool Shen) en tant que rappeurs " authentiques » ou « rappeurs réels » dans l'imaginaire des auditeurs, élargit l'imaginaire de la cité, de la polis en tant qu'espace d'appartenance et d'interaction à un espace urbain international. Si d'autres featuring entre Français et Américains tels qu'ol'kainry feat. Raekwon (2006), Kaaris feat. Future (2015) sont produits, cette même logique de validation, d'authenticité, voire de solidarité entre des vécus perçus comme similaires, réapparaît autour de récits de trafic et la consommation de drogue, l'usage de la violence, et sur le passage ou l'occupation d'un espace pauvre et urbanisé.

Dans un second temps, on remarque que la comparaison entre deux espaces produit un ancrage d'autant plus fort lorsque l'espace de référence est comparé à une institution de prestige. En 2005, le succès du titre " 93 Hardcore » du duo Tandem (C'est toujours pour ceux qui savent, Wagram Music $\left.{ }^{18}\right)$ constitue une véritable introspection géopoétique et musicale du 93. Mac Kregor (Makenzy Guerrier) et Mac Tyer (Socrate Petnga) y font de multiples références à l'institution: " on est pertinemment conscient de tous nos échecs scolaires, mais tout serait différent si la Sorbonne serait [sic] domiciliée à Auber ». La sensibilité de l'auditeur est prise à partie et sa fascination pour le département questionnée : "viens faire un tour dans mon 9-3 si tu l'aimes tellement ». Sur le refrain, le duo crée un call and response sonore avec un collectif d'individus masculins situés " en bas d'la tess ", auquel Tandem se mêle. La clameur " quatre-vingttreize/hard-core" chantée par Tandem et le groupe fonctionne comme une interpellation, elle est l'image et le son d'une identité spatiale et poétique. Aussi, cette structure antiphonique empruntée aux pratiques culturelles afro-diasporiques telles que décrites par Paul Gilroy (2017) est fondamentale, puisqu'elle fait du rap une expérience dans laquelle la hiérarchie entre le son et le sens est abolie, et où l'auditeur se voit recruté par l'écoute participante. Avec cette technologie esthétique et poétique, la performance devient un espace de création de formes, ainsi qu'un lieu de praxis : elle se superpose et se mêle au lieu géographique, elle lui fait écho, elle lui fait désordre, elle amplifie et rend visible l'inscription du politique dans l'espace.

En 2016, le rappeur Fianso (Sofiane Zermani) répond à l'appel de Tandem lors de l'épisode 6 du projet \#JesuispasséchezSo. Ce dernier invite Kalash Criminel sur le morceau " 93 Empire $^{19}$ ", et forme un duo percutant. Cette reprise place " 93 Hardcore » comme un classique du genre hardcore et de l'esthétique sonore du rap du 93, tout comme elle entend reprendre et enrichir le genre. En effet, la série musicale \#JesuispasséchezSo est constituée de onze freestyles de rap filmés dans les grands ensembles de France, définis comme des "endroits de kaïra ${ }^{20}$ ». Optant pour le rôle de l'ambassadeur souverain, Fianso filme des lieux tels que le quartier de la Castellane à Marseille pour mettre en scène sa persona de héros de la street culture. Son gimmick « ish-ish » renvoie au coup de coude du muay-thaï, à l'univers du combat sanglant que Fianso associe à une masculinité déviante appelée « bandit saleté ». Adoptant les traits de la représentation virile, sensationnelle et menaçante produite et imaginée par le traitement médiatique des quartiers populaires en France, Fianso lui donne un visage, une voix, ainsi qu'un plaidoyer. La mise en scène de l'espace et du collectif de \#JesuispasséchezSo utilise le 
potentiel performatif du « mec de cité » face aux trois principes d'ordre, de salubrité et de sécurité publique définis pour gouverner la cité au sens politique et juridique du terme. Dès lors, la rhétorique de Fianso donne à voir et à entendre des espaces habités par des individus impériaux, affranchis, puissants, constituant un "empire» imaginaire. Dans cet « empire » sémantique et sonore, les villes du département du 93 deviennent des institutions du crime, et participent à la renommée du lieu, selon une ghettocentricité assumée. Par exemple, le surnom «Blankok » est donné à la ville du Blanc-Mesnil où Fianso a grandi, et fait référence à la capitale thaïlandaise ayant la réputation d'être une plaque tournante de la drogue. Cette nomination construit un exotisme établi sous le signe de l'illicite, en dehors des lois, tout comme elle élargit la notion d'empire à un territoire dépassant les frontières nationales, linguistiques et culturelles. De plus, l'espace imaginé par Fianso joue sur l'intertextualité musicale et culturelle puisque le refrain de "93 Empire» comporte la punchline de Tandem: "J'baiserai la France jusqu'à ce qu'elle m'aime » (à environ 1'20 des morceaux de Tandem et Fianso). À la manière d'une archive historique et sonore, la citation crée une continuité intergénérationnelle entre les rappeurs, réhabilite et étend l'univers poétique du 93 à travers l'histoire. Avec ce procédé, Sofiane Zermani montre ce qui précède sa performance, ce dont elle se fait l'écho, et se positionne comme passeur. Comme une devise, la punchline désigne un espace national polarisé entre la France des institutions et les quartiers populaires de la Seine-Saint-Denis, contre-espaces situés à l'intérieur du territoire national mais présentés négativement dans le discours public. L'image produite par la punchline "J'baiserai la France jusqu'à ce qu'elle m'aime ", révèle un positionnement contestataire à l'intérieur d'un espace organisé pour abuser le rappeur en tant que corps n'ayant pas droit de cité, de circuler, de prendre la parole, d'exister. De fait, la radicalité de la référence sexuelle s'aligne avec la radicalité de la violence systémique liée à une France qui ne l'aime pas, à une France construite comme un corps doué de mépris. En 2018, Fianso prolonge son geste artistique et critique avec la compilation 93 Empire $^{21}$. Celle-ci marque le succès viral de la série, et réaffirme l'héritage poétique musical hardcore de la Seine-Saint-Denis. Le titre "Sur le drapeau ", en duo avec les Suprême NTM, constitue un autre passage entre deux générations de rappeurs, et esquisse l'idée d'une autonomie politique et culturelle en lien avec la propagation d'affects intenses : «maintenant même le maire connaît le son, je crois qu'il veut même dabber ${ }^{22}$ ». Contagieuse, l'expérience esthétique du rap hardcore est ici décrite comme faisant irruption dans le corps d'un élu de la République. La figure du maire est incorporée à la célébration du contre-espace poétique et culturel, et cette célébration est rendue possible dès lors que les rappeurs du 93, postulant le pouvoir de la musique, réussissent à s'en servir contre les assignations racistes et classistes.

Revendiquer un espace et une appartenance à la polis par une poétique du son et du sens, créer des motifs esthétiques propres à l'histoire d'un lieu marqué par la violence de l'État; telle est l'œuvre des rappeurs du 93, créateurs d'un véritable héritage musical, visuel et textuel hip-hop, et d'une communauté d'artistes intergénérationnelle. 


\section{Le geste : perturber l'ordre, provoquer la justice}

15 En France, une justice indifférente aux violences policières ciblant les jeunes français·es non blanche's habitant les quartiers populaires a mis en évidence l'existence de groupes d'individus traités comme des " citoyen.ne.s de seconde zone ", privés de leurs droits fondamentaux et ce, de manière systémique. La catégorie "jeune" (Maira Sunaina 2014) est ici comprise en tant que signifiant désignant des "sujets en formation" ("subject in the making»), pris dans un contexte social et historique spécifique. Cette catégorie est aussi celle qui cristallise les peurs et les angoisses à propos de la fabrique du sujet national, du débat sur les droits, sur l'appartenance, dans le cadre de la redéfinition d'un nouvel ordre social dans un monde globalisé. Pour ce qui est des rappeurs, Karim Hammou (2018) souligne une certaine réception par laquelle il s'agirait de "traiter ces rappeur.euse.s en étranger.ère.s à la communauté civique, voire en ennemi.e.s intérieur.e.s, et proscrire autant que possible leur participation aux espaces publics médiatiques ». La proscription des rappeurs et rappeuses décrite par Karim Hammou participe à la normalisation d'une mécanique visant à effacer et exclure du débat les discours critiques et dissonants produits par les jeunes artistes et penseurs issus des quartiers populaires. Face à ce déferlement de violence à l'endroit de leurs paroles et de leurs identités, les rappeurs adoptent différentes stratégies rhétoriques et discursives afin de rejoindre le débat par des chemins détournés. Ces stratégies ciblent d'une part les gouvernants et leurs déviances vis-à-vis de la loi, d'autre part, elles sont utilisées comme modes de réflexion critique à propos de la ligne de partage entre le licite et l'illicite, le juste et l'injuste, l'acquisition et le refus des droits. Contre une lecture aveuglément universelle du récit national français, les rappeurs ouvrent les archives orales de l'hégémonie blanche à la française pour mieux questionner les limites de la cohésion nationale en France. Mais la réception de ces pratiques artistiques et engagées dérange au point de propulser les rappeurs et leurs œuvres en dehors du cadre de la performance.

La vidéo du titre "Toka ${ }^{23}$ » (Bandit Saleté, Affranchis Music, 2017) de Fianso cristallise bon nombre de critiques faites aux rappeurs hardcore jugés politiquement incorrects. Dans les faits, son tournage donne lieu à une opération escargot sur l'autoroute A3 au niveau d'Aulnay-sous-Bois (93). Le geste, emprunté à la culture de la revendication et de la manifestation civique en France, est traité comme une stratégie de marketing "salissante " par la journaliste Stéphanie Binet ${ }^{24}$. Si l'intention poétique du geste peut se défendre sur le plan esthétique, la performance artistique - car c'est bien de cela qu'il s'agit-prend des dimensions que seul l'art contemporain semble avoir expérimentées jusqu'ici. "Tout ce que je vous ai volé» d'Hervé Paraponaris (FIAC, 1994), expose des objets du quotidien volés par l'artiste, l'exposition Hardcore: vers un nouvel activisme de Jérôme Sans au Palais de Tokyo (2003), avec des œuvres telles qu'« Instinct de mort » d'Alain Declercq pour laquelle un des policiers ayant abattu Jaques Mesrine réalise un dessin en impact de balles, les œuvres de Banksy, ou encore, les performances de l'artiste palestinien Khaled Jarrar (2015) où ce dernier tire à l'arme à feu, sont autant d'actes défiant la légalité (il est interdit en France, de tirer sans permis ou en dehors du cadre de ses fonctions) mais défendus comme des objets d'art symboliques de la liberté d'expression par les critiques d'art et les médias. Ces cas particuliers montrent comment le caractère artistique d'un geste l'emporte sur le caractère illégal de ce même geste en fonction du contexte de la réception de l'œuvre, 
des auteurs, des lieux de la performance : rues et musées pour Paraponaris, Banksy, Declercq, galerie homologuée pour Jarrar (qui a aussi performé dans des check-points de Palestine) et domaine public routier national et Youtube pour Fianso. Ces exemples d'esthétiques hardcore, et de réceptions d'un geste illégal en art montrent que ce n'est pas le geste artistique mais bien la performance de la persona hardcore de Fianso qui place Sofiane Zermani en infraction, et questionne sensiblement l'espace-temps de l'expérience esthétique du rap. Or, selon Artaud, c'est précisément en ce point où se mêle l'imaginaire, le réel, les effets de style, ainsi qu'un travail sur la matérialité, que l'anarchie fait irruption. Performé dans un espace non homologué, reçu comme une déviance vis-à-vis de la loi, le clip de «Toka » ne pourrait-il pas être lu comme de la " poésie réalisée ", comme un geste contestataire, (à l'image des opérations escargots), opérant la rencontre entre l'imaginaire et le réel? Geste revendicateur d'un droit de cité : celui d'exister dans la polis, et aux yeux et dans les oreilles de la République française, l'exemple de Fianso montre comment le rappeur est projeté dans un spectre éthique polarisé entre la possibilité d'être une influence positive auprès des « jeunes de quartiers", ou celle "d'en être un ", placé en infraction dans ses propos, ses actes, voire, ses relations, son identité, et ce avant même d'être perçu comme poète, artiste ou penseur, non pour autant exempt de critiques. Une forme d'infraction par proxémie, "espace de proximité autour du sujet» (Barthes [1978-1980] 2003: 157), se met en place, dans le sens où l'œuvre du rappeur est constamment jugée en fonction de sa proximité physique avec des lieux et des individus perçus comme indésirables. Ce paradoxe place le rappeur au cœur d'une fantasmagorie de la criminalité associant la personnalité de l'artiste à l'entité fictive, anonyme, dangereuse et anarchique des «jeunes de banlieue». On attend alors du rappeur qu'il compense l'a-moralité de ce fantasme auquel il appartient en ne le confirmant pas, en devenant l'exception qui confirme la règle, méritant donc le statut de poète ${ }^{25}$.

17 Agissant selon des logiques souvent très éloignées de ces assignations morales, les rappeurs hardcore continuent de choquer l'opinion publique en utilisant le potentiel du rap à excéder le cadre de la performance, via un jeu de références et de mise en scène. En guise d'introduction officielle à "Toka », Fianso tweete : «Prendre un café sur l'autoroute, ça n'a pas de prix ${ }^{26}$.» Ouvrant sur un univers où certaines choses n'ont pas de prix, la déclaration du rappeur projette le café, produit emblématique de l'histoire de la plantation, et symbole de la culture du centre de Paris, en dehors de son statut marchand, pour le ramener et l'adapter à un espace en marge, la banlieue parisienne, le périph' étant l'accès indispensable entre les banlieues et le centre parisien. Nous suggérons alors que la rhétorique générée par la vidéo de "Toka » fait écho au concept court-circuitant de «contre-espace public» formulé lors des contestations de Nuit Debout ${ }^{27}$, tout comme elle met en place une certaine praxis, un geste, plutôt qu'une image figée. Aussi, « Toka » marque l'utilisation de la performance de rap hardcore comme outil de résistance à un quotidien structuré par l'ordre et le capital, et rend visible une forme de manifestation critique, considérée comme illégitime dès lors que son sujet est l'habitant non-blanc d'un quartier populaire de France, et que sa forme dépasse le cadre de la loi.

18 Une autre question se pose de fait : comment l'image d'un blocage de l'autoroute peutelle entrer dans le champ poétique? Pour y répondre, une lecture de l'œuvre en tant que système référentiel est nécessaire. Le refrain du morceau produit une entité collective dont les attributs sont le pistolet Tokarev TT 33 et l'image sonore de la manchette («ish ish»). Sa sémantique projette l'auditeur dans une spatialité figurée par 
trois villes de trois continents distincts : « l'équipe est toka, ish ish / Alger Paris Bagdad, ouais ». Ici, la revendication spatiale crée une liaison symbolique, une systémique affectées entre les trois capitales. Les armes corporelles (le coup de coude) et matérielles (le Tokarev), suggèrent une violence faite au corps ou produite par le corps. Alger et Bagdad étant des lieux sujets à l'occupation occidentale, la ville de Paris est projetée dans l'espace-temps de la guerre, prise à partie dans sa relation aux armes, à sa souveraineté politique et diplomatique, et à ses droits vis-à-vis des autres espaces, sa participation aux conflits internationaux et à son passé colonial. La combinaison entre un imaginaire décentré, ouvert sur l'extérieur et le lieu de l'autoroute - voie rapide servant à l'exploitation et à la pénétration de l'espace - recrute la subjectivité des auditeurs dans un ordre anarchique où les capitales d'un monde en guerre ne sont plus que des points d'ancrage, des espaces d'accumulation d'une violence normalisée, voire souveraine, auxquels mènent tous les chemins. Dans ce monde dystopique, l'appréhension et l'articulation de la violence par la persona de Fianso, ici épique, lui confère un savoir-faire à toute épreuve, une lucidité singulière, une aura édifiante.

Puisque " provoquer la justice » peut s'entendre soit comme une perturbation à l'ordre, soit comme un désir de justice, cette lecture alternative et poétique du geste de Fianso, capturé et mis en scène dans le clip de "Toka », ne cherche pas simplement à offrir une analyse critique du rap au travers de ses dimensions sonores, visuelles, et esthétiques, mais elle vise aussi à faire valoir la performance de rap comme un objet à part entière, sujet à de multiples lectures aussi originales que singulières. Enfin, cette lecture interroge moins les intentions du rappeur qu'elle ne se focalise sur la réception et l'écoute de son œuvre.

\section{Le feu : signaler l'injustice, rectifier l'histoire}

La création de personae à partir d'identités sur lesquelles se focalisent les peurs sociales assigne les rappeurs à succès à une position de porte-parole lorsqu'il s'agit de traiter des quartiers populaires en tant que tels dans le débat public. Le 11 février 2017 à Bobigny, Sofiane Zermani use de sa notoriété pour éviter l'affrontement lorsque des policiers menacent de disperser la manifestation en soutien à Théo Luhaka. Cette stratégie dévoile le potentiel d'un engagement aux effets manifestes. Si le style hardcore place le corps et l'intime au centre de la performance, nous l'avons vu, cette poétique du corps est associée à une critique du politique, de l'histoire et de leur violence respective. Une pensée mêlée d'affects intenses est mise en circulation via la musique de rap hardcore. Les manques et les absences de l'histoire sont reconnus, épinglés, discutés dans des morceaux de rap offrant un vocabulaire et des formes pour penser l'injustice, les identités raciales politisées, et pour développer des critiques. Cette position d'intellectuel, avec la musique pour outil de pensée, est analysée par le rappeur Médine lorsqu'il désigne la dimension militante de son rap. En 2017, il explique au journaliste Mouloud Achour :

J'utilise cette musique, je la conçois comme un moyen d'éducation populaire, comme quelque chose qui peut nous permettre de nous émanciper, comme un canal d'information clairement, et lorsqu'on vient me questionner sur des sujets périphériques et qu'on oublie même cette musique, ça me dérange, mais aujourd'hui je l'assume, et j'ai jamais refusé une discussion avec un média ${ }^{28}$.

La conception du rap par Médine rejoint l'idée de l'art comme " espace de symbioses » où « le problème posé à l'artiste n'est pas le sien, il est celui de la collectivité et voilà 
pourquoi, s'il est bien le seul créateur de l'œuvre, celle-ci a pour auteur la communauté qui l'accomplit» (Diagne 2007: 99). Cette conception permet de penser ensemble la persona du rappeur, son identité, son corps, ainsi que la communauté à laquelle il se réfère. Lorsque Médine regrette que l'aspect musical de son œuvre soit mis de côté, c'est que selon lui, le rap peut être une philosophie, un mode de connaissance en soi, et ce, au travers de ses formes textuelles, visuelles, sonores et poétiques. En tant qu'espace de diffusion de la mémoire de la violence faite aux corps, le rap amorce une réflexion authentique sur le sujet humain, les formes de vie ébranlées par la répression $\mathrm{du}$ pouvoir. Le rap produit du savoir, et la dimension intellectuelle du rap fait signe vers une écoute engagée. Cette écoute informe l'auditeur des débats de la communauté hip-hop locale, globale, des débats liés à l'histoire des luttes afro-américaines ainsi qu'à l'Atlantique noir, l'histoire de la plantation, la colonisation, et celle de l'immigration en Europe. Cette dimension politique trouve son motif dans la figure de "l'anarchiste couronné » d'Artaud, qui rappelons-le, est un souverain utilisant la poésie pour gouverner la polis, et déployer d'autres approches du politique.

Pour comprendre comment s'articule la dimension du poétique, du politique et du sensible, l'architecture à huit voix du morceau «Grand Paris ${ }^{29}$ » de Médine (Prose Élite, Din Records, 2017) est emblématique, puisqu'elle propose un espace où les rappeurs sont aussi les auditeurs. Le beat produit par Proof est composé d'un carillon bourdonnant et grésillant, passé à l'effet « sous l'eau » et connotant des affects à la fois mélancoliques et palpitants. Les flows de Médine, Sofiane et Youssoupha sont staccatos, et contrastent avec le tempo plus relâché des passages de Lartiste, Lino, Alivor, Seth Gueko et Ninho. Chacun des passages aborde la pratique du rap, les violences policières, l'histoire de France, le genre, l'islamophobie, la race, la sexualité, l'espace urbain local, national, extra-national. La répétition collective de la revendication «C'est nous le Grand Paris » crée une communauté tissée entre les différentes identités des rappeurs. $\mathrm{Si}$ « le Grand Paris » fait référence à la politique de la ville lancée par Nicolas Sarkozy en $2007^{30}$, l'entité ouvre ici sur un espace nouveau, traversé par des sonorités hip-hop, tourné vers l'histoire des corps. Bien que Médine, Proof et Alivor soient originaires du Havre, la polyphonie vocale ainsi que les différentes géo-identités invoquées sur le morceau matérialisent un Grand Paris du rap. Cette architecture sonore et poétique vient alors étendre et confondre les limites de l'espace : plus qu'un jeu sur les personae, les identités culturelles sont investies par le biais de marqueurs spatiaux. Le style hardcore articule l'idée d'une parole soutenue par une expérience vécue, d'un rapport intime au lieu, et d'une violence esthétisée, redirigée vers l'espace politique duquel elle provient. La proéminence des références aux axes de transports tels que "l'artère " parisienne, l'autoroute ou le port, suggère un Grand Paris mobile, constitué de manière organique et non fixe. Ces mouvements incessants opèrent une extension significative de la revendication spatiale puisque la mobilité des sujets convoque une approche phénoménologique de l'espace incluant notamment l'identité racialisée.

On peut s'aider de la phénoménologie queer de Sara Ahmed (2007) pour comprendre cette relation intime et performative entre le corps et l'espace. La philosophe montre que le corps pensé dans sa surface prête sa peau à l'espace social, alors formé et modelé par la répétition et la multiplicité du passage des corps dans cet espace. Dès lors, les modes d'opération du corps sont structurés selon un certain schéma racial, puisque le monde hérité précède le point d'arrivée du corps dans l'espace, et que la construction sociale de la race se forme autour d'un passé hérité et matérialisé à même le corps. 
Pour Sara Ahmed, la peau de l'institution au passé colonial est blanche, et l'espace se perçoit comme un ready-made pour le corps blanc puisque, sur le mode de la ressemblance, «l'espace étend les corps et les corps étendent l'espace» («So space extends bodies and bodies extend spaces ») (Ahmed $2007: 158$ ). La phénoménologie de Sara Ahmed nous montre que le principe de la corporalité du pouvoir se déploie selon des lignes de ressemblances ne se limitant ni à la race ni au genre, et touchant les corps dans leur ensemble. Cette co-construction de l'espace attribue une extension à certains corps et pas à d'autres; elle nous fait comprendre comment certains corps se fondent et passent aisément dans l'espace, tandis que d'autres provoquent une perturbation et se déplacent difficilement dans l'espace. Avec la pensée d'Ahmed, les références à l'espace et à la mobilité se chargent d'une couche philosophique et critique. La dichotomie de l'artère employée par Médine et Lartiste se comprend alors comme une réappropriation corporelle et historique de l'espace public parisien : « Tu vois pas qu'ils veulent voir Paris par terre? Pas tant qu'on traînera dans ses artères, pas tant qu'on traînera dans ses artères. » La charge sensible et poétique placée sur la capitale désigne précisément ce passé hérité des violences coloniales et policières ayant contribué, et faisant perdurer dans le présent, via la gentrification notamment, la formation d'un espace où le mouvement des corps non conformes à la blanchité dominante est mis en difficulté. Scrutant ce passé hérité dans le but d'en inverser les effets dévastateurs, Médine imagine la panthéonisation de Zyed Benna et Bouna Traoré, deux adolescents tués par harcèlement ${ }^{31}$ policier à la suite d'un contrôle le 27 octobre 2005 à Clichy-SousBois. Ses rimes incisives tendent à rétablir une mémoire du lieu et des corps interpellés du fait de leur non-ressemblance à la peau de l'espace social : « dès que le cœur d'un grand homme s'arrête / Paris donne son nom à une artère / moi je suis pour que le boulevard de la Villette / soit rebaptisé Bouna \& Zyed». Cette démarche enclenche également un déblanchiment de l'espace, et ce dans l'espoir de normaliser la trajectoire des corps qui ne s'y fondent pas. Elle amorce une reconquête du droit de cité pour ces corps, pour les rappeurs et leurs auditeurs.

Outre les corrections apportées à une lecture institutionnelle de l'histoire, le refrain du morceau «Grand Paris » cherche à légitimer l'espace de la banlieue : «la banlieue influence Paname, Paname influence le monde ». La formule fait l'objet de répétitions et variations, et place le signifiant « Paname » entre la «banlieue », le « 93 », « 91 », les «kaïras", Chicago, l'Afrique, le Maghreb et le monde. Selon les paroles de Lino, les identités plurielles des quartiers populaires du Grand Paris sont disséminées par les corps dans l'espace, pour en devenir le cœur battant: "on ne stoppera pas l'hémorragie banlieusarde ». La sémantique du sang s'échappant des corps, ainsi que l'utilisation de l'adjectif "banlieusard », renvoie à une pluralité, voire à un désordre, pour reprendre Artaud. Là où aurait pu se trouver la traduction d'un concept d'identité hybride entre le fait de posséder un héritage de culture étrangère et française, la culture «banlieusarde» fait imploser cette logique binaire, dessine une identité complexe et opaque, sans limites géographiques concrètes, une identité prenant la forme d'un collier de perles. En effet, en cherchant à habiliter les corps non blancs dans l'espace de la capitale française, le morceau "Grand Paris » de Médine fait référence à une identité française et «banlieusarde » et étrangère, qui plus est déployée et définie au travers d'une polyphonie rappée : « la banlieue influence Paname, Paname influence le monde ». L'exploration poétique et musicale de l'identité du collectif " Grand Paris » suppose une approche transversale et affectée des frontières; elle récrée une polis poétique, rend possible la critique du sentiment d'appartenance à la nation, ainsi que le 
débat sur la souveraineté nationale derrière lequel se cachent certains sujets qui en excluent d'autres en supprimant leurs droits de cité. En tant que tel, l'espace musical et discursif du morceau abrite une contre-métropole décoloniale et poétique hardcore tout comme il concrétise la dimension intellectuelle et militante du rap.

\section{Conclusion}

La souveraineté du rappeur et le droit de cité du rap s'arrachent par la force des mots, par la puissance du son. Ce droit est avant tout un droit à une existence légitime et souveraine, à l'intérieur d'un espace dénié aux communautés d'individus laissés pour compte et mis à pied par une histoire et un système de loi dont les manquements, les silences et les oublis blessent et tuent. En rappelant que par proxémie, le premier public du rap est constitué d'auditeurs issus des quartiers populaires, le mythe associant l'imaginaire de la banlieue, le fantasme de l'émeute, l'idéal révolutionnaire, et le rap comme arsenal de cette rhétorique, a de beaux jours devant lui. L'autre réalité du rap et de ses performeurs est que cette musique subit les préjudices et injustices que la police et la justice réservent aux quartiers populaires de France. Dès lors, l'intérêt mutuel des uns pour les autres poursuit un même but de respectabilité. Penser le rap et sa poétique en lien avec le politique permet de dévoiler les effets particuliers du rap à l'endroit du corps, puisque ce dernier devient l'objet et le sujet de la performance hardcore. Le corps est d'une part, le support de la persona du rappeur, et d'autre part le lieu où les affects font surface, avant de se mêler à l'imaginaire et à la pensée. La notion d'espace est alors comprise dans sa dimension sensible, corporelle, phénoménologique et la musique, de par sa structure vibrante, accorde le corps et le sujet, et les oriente dans l'espace. Le sujet-corps pris dans son positionnement au monde revendique la spatialité qu'il habite, et le rap est le moyen de penser l'identité comme ce qui prend forme par l'occupation d'espaces géographiques, à travers le temps, ce qui prend forme dans l'épaisseur des corps, et au travers du signifiant racial. Dans l'imaginaire du rap hardcore, l'espace de la cité est le terrain d'une bataille sémantique déterminante, où les barrières sociales, matérielles et politiques sont déconstruites, où l'ordre est malmené. Les performances de rap y voient tellement juste qu'elles se heurtent aux lois concrètes de la polis qui, ayant fini par les craindre, s'obstine à restreindre le rap à ses clichés. Enfin, la réalisation collective de la critique hardcore d'un monde violent fait imploser le concept hégémonique de souveraineté nationale, d'une communauté d'ayant-droit sur les autres, dans une république excluant les corps étrangers, non blancs, pauvres, sexisés, tout en prenant à parti une morale bourgeoise et conservatrice. Cette critique s'attaque à l'altérisation des cultures étrangères, réhabilite la souveraineté de ces Autres, dépouillée et ignorée par des récits historiques erronés, aporétiques. Elle sert une cause et un plaisir communs, de voir se réaliser de multiples possibles dans des imaginaires affranchis, dans des corps battants. Intense et saturée, faite "de cri, de sang, de geste et de feu", la performance hardcore qui provoque une explosion d'affects jusque dans la chair, fait des rappeurs et de leurs auditeurs des sujets libres et souverains, des anarchistes couronnés. 


\section{BIBLIOGRAPHIE}

Ahmed, Sara, 2006, Queer Phenomenology: Orientations, Objects, Others, Durham, Duke University Press.

Ahmed, Sara, 2007, « A phenomenology of whiteness », Feminist Theory, vol. 8, nº 2, p. 149-168.

Artaud, Antonin, [1979] 2007, Héliogabale ou l'Anarchiste couronné, Paris, Gallimard.

Baldwin, Davarian L., 1999, « Black Empires, White Desires, The Spatial Politics of Identity in the Age of Hip Hop », Black Renaissance Noire, vol. 2, nº 2 .

Barthes, Roland, 2003, La préparation du roman, Cours au collège de France (1978-1979 et 1979-1980), Paris, Seuil.

Bourdieu, Pierre, 1996, Sur la télévision, suivi de l'emprise du journalisme, Raisons d'Agir.

Dalibert, Marion, 2018, « Les masculinités ethnoracialisées des rappeureuse·s dans la presse », Mouvements, nº3, p. 22-28, [En ligne], https://doi.org/10.3917/mouv.096.0022.

Dalibert, Marion, 2020, «Le métarécit national des médias d'information : entre production de la race et de la classe et légitimation des rapports sociaux ", Recherches féministes, $n^{\circ} 33$, p. 35-51, [En ligne], https://doi.org/10.7202/1071241ar, [dépôt version auteur], https://hal.archivesouvertes.fr/hal-02943206.

Derrida, Jacques, 1967, L'écriture et la différence, Paris, Seuil.

Diagne, Souleymane-Bachir, 2007, Léopold Sédar Senghor : l'art africain comme philosophie, Paris, Riveneuve.

Gilroy, Paul, 2017, L'Atlantique noir : modernité et double conscience, trad. Charlotte Nordmann, Paris, Éditions Amsterdam.

Grassy, Elsa, 2010, Le Lieu musical : du texte à l'espace, un itinéraire sémantique. Poétique des catégories géographiques dans les musiques populaires américaines (1920-2007), Thèse de doctorat sous la direction de M.-M. Martinet, Université Paris IV Sorbonne.

Guillard, Séverin, 2017, « "Getting the city on lock" : imaginaires géographiques et stratégies d'authentification dans le rap en France et aux États-Unis ", L'Information géographique, vol. 81, $\mathrm{n}^{\circ}$ 1, p. 102-123, [En ligne], https://doi.org/10.3917/lig.811.0102.

Guillaumin, Colette, [1972] 2002, L’idéologie raciste : genèse et langage actuel, Paris, Gallimard.

Hall, Stuart, 1989, «Cultural Identity and Diaspora », Framework: the Journal of Cinema and Media, $\mathrm{n}^{0} 36$.

Hammou, Karim, 2018, « Rap en France et racialisation, Entretien avec Karim Hammou, réalisé par Patrick Simon », Mouvements, n 96, p. 29-35, [En ligne], https://doi.org/10.3917/mouv. 096.0029.

Hebdige, Dick, 1979, Subculture: The Meaning Of Style, Londres, Routledge.

Khiari, Sadri, 2006, Pour une politique de la racaille : immigrés, indigènes, et jeunes de banlieues, Paris, Textuel.

Kitwana, Bakari, 2005, Why White Kids Love HIP-HOP, wankstas, wiggers, wannabes, and the new reality of race in America, New York, Basic Civitas Books. 
LaBennett, Oneka, 2011, She's Mad Real: Popular Culture and West Indian Girls in Brooklyn, New York, New York University Press.

Miller, Matt, 2004, « Rap's Dirty South: From Subculture to Pop Culture », Journal of Popular Music Studies, $\mathrm{n}^{\circ}$ 16, p. 175-212, [En ligne], https://doi.org/10.1111/j.0022-4146.2004.00018.x.

Rolnik, Suely, [2011], Anthropophagie zombie, traduit du portugais (brésil) par Renaud Barbaras, Blackjack éditions.

Rose, Tricia, 1994, Black noise: Rap Music and Black Culture in Contemporary America, Hanover, Wesleyan University Press.

Saïd, Edward, [1978] 2015, L'orientalisme. L'Orient créé par l'Occident, Paris, Seuil.

Sédel, Julie, [2009] 2013, Les médias et la banlieue, Préf Gérard Mauger, et Postface Patrick Champagne, éd. Le bord de l'eau.

Sunaina, Maira, 2014, « Youth », dans B. Burgett et G. Hendler (dir.), Keywords for American Cultural Studies, New York, Londres, New York University Press, p. 245-248.

\section{NOTES}

1. Nous remercions Meïla Assani pour ses précieux conseils, ses compléments d'analyse et son soutien. Voir son travail de mémoire, «Les stratégies identitaires développées par les NTIC : l'émergence d'une subculture afropéenne francophone grâce aux NTIC », (2018) http://hdl.handle.net/2078.1/thesis:14530.

2. Nous remercions Jean-Pierre Seck, Driver et l'équipe du média La Récré pour leurs suggestions.

3. La punchline de Médine "I am muslim, don't panic » est par exemple popularisée grâce à la vente de $t$-shirts par le rappeur.

4. Se dit d'une œuvre de fiction privilégiant les scènes sanglantes.

5. Pour une lecture typiquement issue des cultural studies, voir la notion de culture hégémonique dans Stuart Hall, «Identité et culture, politique des cultural studies ", 2007, p. 421-422.

6. Le droit de cité définit l'ensemble des privilèges qui étaient attachés à la qualité de citoyen d'une cité antique. Dans la pensée moderniste, l'expression droit de cité désigne un ensemble de droits civiques, tel que le droit de vote. L'expression "avoir droit de cité", elle, est issue du monde militaire, et désignait l'autorisation donnée à des militaires pour défiler dans la ville avec un attirail, ou encore le fait d'avoir "les clés de la ville". Dans le langage courant et en littérature, cette expression désigne le fait d'être admis ou exclu d'un espace, notamment, un espace délimité politiquement, tel que, une nation, une démocratie, une république, une ville et ses habitants. Cette expression française n'a d'ailleurs pas de traduction exacte en anglais. Elle se traduit par le terme citizenship, qui désigne la citoyenneté et l'appartenance à une nation.

7. Selon le chercheur Matt Miller (2004), cette région est aussi désignée comme la Third Coast. Développée par les rappeurs d'Atlanta, la trap music est reconnaissable par ses sonorités lugubres, des basses ultra saturées, un rythme lent, une utilisation minimaliste de l'instrument, ainsi qu'un univers inspiré d'une forme diabolique du Hoodoo afro-américain. Ce style est popularisé en France par le rappeur sevranais 
Kaaris avec le titre «Zoo » d'Or Noir ([disque audio, téléchargement digital], Universal, Capitol, AZ, Therapy Music, 21 octobre 2013).

8. Yard, "Kalash Criminel : "Il y a de la violence partout, pas que dans le quartier" ", YouTube, 4 mai 2016, [En ligne], https://youtu.be/kbM0SLJU0q4 (consultée le 2 juillet 2021).

9. Kalash Criminel, «Coltant », La Fosse aux lions [disque audio, téléchargement digital], Sales Sonorités, 23 novembre 2018, 18 titres, $1 \mathrm{~h}$.

10. Yard, "Kalash Criminel : "Il y a de la violence partout, pas que dans le quartier" ", op. cit.

11. Rapelite.com, « Kalash Criminel : sa mixtape, 1012 14, Kaaris, le foot, RDC 243, De La Villardière à Sevran, la fac », YouTube, 11 octobre 2016. À 6'30 minutes, [En ligne], https://youtu.be/J-TgV6i2qbE (consulté le 2 juillet 2021).

12. Kalash Criminel, "Cougar Gang », YouTube, 9 novembre 2018, [En ligne], https:// youtu.be/kyfWdawFxZk (consulté le 2 juillet 2021).

13. Team Mouv', «Kalash Criminel, son "Cougar gang” censuré suite à des pressions de l'Élysée », Mouv', 15 novembre 2018, [En ligne], https://www.mouv.fr/rap-fr/kalashcriminel-ete-censure-par-l-elysee-pour-son-cougar-gang-345750 (consulté le 2 juillet 2021).

14. Sur les rapports entre une subculture et le pouvoir, voir les travaux de Mike Brake, The Sociology of Youth Culture and Youth Subcultures, 1980.

15. Sur la question de la représentation des Banlieues Rouges ouvrières et de l'immigration, voir, entre autres, les travaux de Sadri Khiari, Pour une politique de la racaille: immigrés, indigènes, et jeunes de banlieues (2006), ainsi que l'étude de Julie Sédel, Les médias et la Banlieue (2009). Pour comprendre la formation d'un espace altérisé par le racisme, nous utilisons L'idéologie Raciste, genèse et langage actuel de Colette Guillaumin ([1972] 2002). Dans les préliminaires de l'ouvrage, elle explique les présupposés centraux de la formation de l'altérité en France : «Pour l'opinion publique, la question du racisme se pose selon deux axes simples, d'une part l'existence d'une différence de "nature" entre des groupes humains, et d'autre part l'exercice de l'hostilité entre ces différents groupes." Elle insiste sur le fait que "la race apparaît [comme] forme biologique utilisée comme signe ", et que ce « système de signe commun [est] médiatisé sur des registres différents" (60). L'importance des médias dans les représentations sociales de la race, de la peur de l'autre est donc centrale, les banlieues parisiennes, en particulier la Seine-Saint-Denis, subissent un traitement altérisant de la part des médias nationaux. De nouveaux médias comme Bondy Blog, StreetPress ou Mediapart tentent aujourd'hui d'inverser cette tendance.

16. Nas, Foxy Brown, AZ, Comega, «Affirmative Action », It Was Written [disque audio], Columbia Records, 2 juillet 1996, 1 CD, 58,20 min.

17. Suprême NTM, Paris sous les bombes [disque audio], Epic Records, 28 mars 1995, 1 CD, $1 \mathrm{~h} 08$.

18. Tandem, « 93 Hardcore », YouTube, 2005, [En ligne], https://youtu.be/icgYS_u8TtI (consulté le 2 juillet 2021).

19. Fianso, Kalash Criminel, " 93 Empire », Je suis passé chez So, épisode 6, [En ligne], YouTube, 8 juillet 2016, https://youtu.be/ThoUcYaQCBc (consulté le 2 juillet 2021). 
20. "Dans tous les endroits de kaïra j'ai laissé mes empreintes », rappe Fianso dans l'épisode 11 de \#Jesuispasséchezso, [En ligne], YouTube, 27 janvier 2017, https:// youtu.be/sGSfN3i4NoI (consulté le 2 juillet 2021).

21. Fianso, Diias, Zeg P, Nock-Pi, Yasser Beats, 93 Empire, [disque audio, téléchargement digital], Affranchis Music, 5 octobre 2018, 1 album, 1 h 17.

22. Le dab est un geste inventé par le basketteur Dee Brown en 1991, il consiste à mettre la tête dans le pli du coude et tendre les bras parallèles aux côtés opposés. Il est utilisé comme une marque de fierté et d'accomplissement notamment dans les événements sportifs.

23. Fianso, «Toka », YouTube, 7 avril 2017, [En ligne], https://youtu.be/XZBU7BWQsZs (consultée le 2 juillet 2021).

24. Stéphanie Binet, "Enquête après que le rappeur Fianso a bloqué l'A3 sans autorisation », Le Monde, [En ligne], 12 avril 2017, https://www.lemonde.fr/musiques/ article/2017/04/12/enquete-sur-l-operation-escargot-du-rappeur-

fianso_5109871_1654986.html (consulté le 2 juillet 2021).

25. Dans l'article de Marion Dalibert, «Les masculinités ethnoracialisées des rappeureuse's dans la press» (2018), la chercheuse décrit la mise en avant de rappeur'euse's perçu'e's comme des contre-exemples d'une certaine norme définissant la catégorie des rappeureuse's comme des hommes non-blancs, homophobes et sexistes. La masculinité des rappeuses est mise en avant de manière ambivalente, allant à l'encontre de l'image d'une femme qui serait libre car féminine, mais aussi, dominée car hypersexualisée.

26. \#Fianso, «Prendre un café sur l'autoroute ça n'a pas de prix », Twitter, 6 avril 2017, https://twitter.com/Fianso/status/850053398871367681 (consulté le 2 juillet 2021).

27. Anonyme, «Il n'y aura pas d'élection présidentielle », Lundi Matin, 5 septembre 2016, [En ligne], https://lundi.am/IL-N-Y-AURA-PAS-D-ELECTION-PRESIDENTIELLE (consulté le 2 juillet 2021).

28. Clique TV, «Clique x Médine », YouTube, 24 février 2017, [En ligne], https:// youtu.be/CXoeEJOD7GQ (consulté le 2 juillet 2021).

29. Médine, Youssoupha, Lino, Lartiste, Fianso, Alivor, Seth Gueko, Ninho, «Grand Paris ", YouTube, 27 février 2017, [En ligne], https://youtu.be/6sfVkZYIyik (consulté le 2 juillet 2021).

30. En tant que grand plan urbain, le « Grand Paris » vise à étendre l'organisation de la capitale française en cherchant à fluidifier tout en repoussant à la périphérie le flux de main d'œuvre et de salariés. Les effets directs de ce plan urbain sont la gentrification des quartiers populaires de Paris et de sa proche périphérie, et l'allongement du trajet des travailleurs les plus précaires. Voir par exemple: Scopfair, "La métropole en guerre contre les quartiers populaires », [billet], 20 septembre 2019, [En ligne], https:// blogs.mediapart.fr/scopfair/blog/200919/la-metropole-en-guerre-contre-les-quartierspopulaires.

31. Le 27 octobre 2005 à Clichy-sous-bois, Zyed, Bouna et Mihittin sont poursuivis par des policiers. Ils se réfugient dans un transformateur éléctrique. Seul Mihittin survit. Le décès des deux adolescents, déclencheur des révoltes dans les quartiers populaires de France, est selon nous, symptomatique du phénomène de harcèlement policier. En effet, la systémique raciste (La mécanique raciste, Pierre Tevenian, 2017), le contrôle répété et abusif des habitants des quartiers populaires, les violences physiques et 
verbales, fonctionnent comme un système d'oppression face auquel les personnes ciblées réagissent par la fuite, entre autres. Le contrôle en tant que tel est bien entendu autorisé par la loi, c'est lorsqu'il est répété, abusif, et violent, qu'il devient illégal. En l'absence d'études prouvant l'inexistence d'un tel système d'oppression, nous nous appuyons sur les études existantes (telle que La domination policière : une violence industrielle de Mathieu Rigouste, 2012), les témoignages et l'observation du réel, bien que comprenant une part de subjectivité liée à la position de l'auteur, pour affirmer que c'est le harcèlement policier, avec le contrôle policier en tant qu'acte abusif, qui a causé la mort de Zied et Bouna.

\section{RÉSUMÉS}

Cet article utilise la figure de l'anarchiste couronné d'Antonin Artaud pour comprendre la poétique du rap français hardcore. Les formes matérielles et sonores de la performance sont analysées, ainsi que la construction de la figure (persona) de rappeur hardcore. On tente alors de comprendre comment le rap hardcore transmet des affects intenses et propose une certaine critique politique au travers de références à l'institution, aux identités et à l'histoire de France.

This article uses Artaud's "crowned anarchist" figure to understand the poetics of French hardcore rap. The sound and the form of the performance, alongside the construction of a hardcore persona are under study. We will then try to understand how hardcore rap forgrounds intense affects, and a certain critique of French politics through references to identities, institutions and French History.

\section{INDEX}

Keywords : hardcore, rap, poetics, identity, performance, politics

Mots-clés : hardcore, rap, poétique, identité, performance, politique

\section{AUTEUR}

\section{ELSA VALLOT}

University of Southern California, Comparative Studies in Literature and Culture Department 